\title{
Giresun-Karagöl Yaylası Karayosunlarında Kurşun Kirliliğinin Bir İncelemesi
}

\author{
Bahadır KOZ
}

\section{$\ddot{\mathbf{O z}}$}

Bu çalışmanın amacı, Giresun-Karagöl yaylasındaki karayosunlarında kurşun analizi gerçekleştirmek, bölge havasının kurşun kirliliği açısından bir değerlendirilmesini yapmaktır. Bu amaç doğrultusunda bölgeden 17 tane karayosunu numunesi toplandı. Toplanan numunelerin tür teşhisleri, çeşitli flora kitapları yardımıyla gerçekleştirildi ve Etüv'de

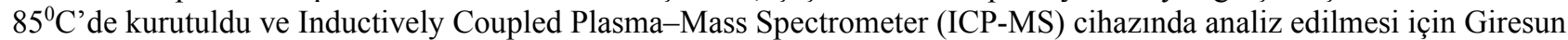
Üniversitesi Merkezi Araştırma Laboratuvarına numuneler gönderildi. Analiz sonucu Kurşun ortalamas $14.47 \mathrm{mg} / \mathrm{kg}$ belirlendi. Bu ortalama, Türkiye'de şehir bölgelerindeki yapılan çalışmalardan elde edilen kurşun ortalamalarının oldukça altında olduğu tespit edildi. Numunelerdeki kurşun miktarının Türkiye'de ve Dünyada yapılmış çalışmalardaki kurşun miktarları ile kıyaslaması yapıldı. Elde edilen sonuçların ekosistem ve insan sağlı̆̆ı üzerindeki muhtemel etkileri tartışıldı.

Anahtar Kelimeler: Ağır Metal, ICP-MS, Karayosunu, Giresun, Karagöl yaylası.

\section{An Investigation of LeadPollution in Giresun-Karagöl PlateauMosses}

\begin{abstract}
The aim of this study is to carry out lead analysis in the mosses in Giresun-Karagöl plateau and to make an evaluation of the air in the region in terms of lead pollution. For this purpose, 17 moss samples were collected from the region. The species identification of the collected samples was carried out with the help of various flora books and dried in an oven at $85^{\circ} \mathrm{C}$ and samples were sent to Giresun University Central Research Laboratory to be analyzed in an Inductively Coupled Plasma-Mass Spectrometer (ICP-MS) device. As a result of the analysis, the average Lead was determined to be $14.47 \mathrm{mg} / \mathrm{kg}$. This average is the average obtained from studies conducted in the lead of cities in Turkey were found to be significantly below. In Turkey, the amount of lead in the samples and performed a comparison with the amounts of lead in studies conducted around the world. The possible effects of the obtained results on the ecosystem and human health were discussed.
\end{abstract}

Keywords: Heavy Metal, ICP-MS, Mosses, Giresun, Karagöl plateau. 


\section{Giriş}

Atmosferik ağır metallerin birikimini ölçmek amacıyla karayosunlarını kullanma fikri, 1960'ların sonlarında (Ruhling ve Tyler, 1968) tarafından geliştirilmiştir. Özellikle halı şeklindeki karayosunları besinlerinin çoğunu çökeltme ve kuru birikim olarak elde ederken, bulundukları substrat'dan çok az metal alımı vardır. Karayosunu analizi tekniği, diğer analiz sistemlerine göre daha ucuz ve daha verimli bir sistemdir (Berg ve Steinnes, 1997).

Hava kirliliğinin monitörlenmesi son 30-40 yılda giderek önem kazanmıştır. Hava kirliliğin teknik ölçümleriyle ilişkili yüksek maliyetlerden dolayı, alternatifler geliştirilmiştir. Biyomonitörleme, kullanılarak biyosferin muhtevasındaki elde edilecek bilgi için doğal metoloji ve verim Wolterbeek tarafından teklif edildi (Wolterbeek, 2002). Böyle çalışmalarda kullanılan karayosunlarının gerçek manada kök, gövde ve yaprakları olmaması dolayısıyla hassas biyoindikatörler olarak bazı avantajlara sahiptir. Ayrıca Karayosunlarının, Yapraklarında kütikül ve epidermis’te yoktur, bu nedenle hücre içerisine ağır metal iyonları kolayca nüfuz edebilir (Grodzinska ve Szarek-Lukaszewska, 2001).

Araçlardan çevreye verilen sadece akaryakıtta bulunan kurşun değil, aynı zamanda, motor yağlanması ve motor gövdesi, araba gövdesi, fren yapma ve lastik aşınmalarından kaynaklanan emisyonların da göz önünde bulundurulması gerekir (Zechmeister ve ark., 2003).

Trafikten kaynaklanan hava kirliliğinde öncelikle belirlenmesinde $\mathrm{Pb}$ (Kurşun), emisyonları çalış1lmıştır (Jaradat ve ark., 1999). Amerika Birleşik Devletleri ve Kanada’da 1990 yılından itibaren araçlarda kurşunsuz benzin kullanma zorunluluğu getirilmiştir (Berg ve ark., 1997, Ruhling ve ark., 1984, Masadeh ve ark., 2002). Türkiye'de de 2007 yılından itibaren kurşunsuz benzin kullanılmaktadır (URL-1).

$\mathrm{Bu}$ çalışmanın amacı, Giresun ili Karagöl yaylasındaki karayosunlarında $\mathrm{Pb}$ (Kurşun) miktarlarını belirleyerek bölgenin kurşun kirliliği bakımından durumunu ortaya koyabilmektir. Bu amaç için bölgeden 2019 Yaz döneminde toplanan 17 karayosunu numunesi önce Giresun Üniversitesi Biyoloji Laboratuvarına getirilip çeşitli Flora kitapları yardımıyla Tür teşhisleri gerçekleştirildi ve daha sonra Ion Coupled Plasma-Mass Spektrometre'si (ICP-MS) cihazında analiz edildi. ICP-MS cihazından elde edilen sonuçlar Literatürdeki diğer çalışmalarla kıyaslandı, elde edilen sonuçlar, insan sağlı̆̆ı ve ekosisteme etkileri açısından tartışıldı. 


\section{Materyal ve Metot}

\section{1. Çalışma Alanı}

$40^{\circ} 07$ ve $41^{\circ} 08$ kuzey enlemleri ile $37^{\circ} 50$ ve $39^{\circ} 12$ doğu boylamları arasında yer alan Giresun İli, Türkiye'nin Doğu Karadeniz bölgesinde yer almaktadır. Doğuda Trabzon ve Gümüşhane illeri, güneydoğuda Erzincan, güney ve güney batıda ise Sivas ve batıda da Ordu ili ile komşudur, kuzeyde ise Karadeniz bulunmaktadır.

Karadeniz kıyısına paralel uzanan Giresun Dağlarından dolayı, dağların kuzey kısımlarında Karadeniz iklimi görülürken, dağların güney kısımlarında ise kısmen Karadeniz ve kısmen karasal iklim görülmektedir.

Karagöl yaylası, İnsan kaynaklı kirlilikten uzak olup yaz aylarında insanların hayvanlarını otlatmak amacıyla çıkarttığı bir yayladır. Bölgede herhangi bir sanayi tesisi veya maden gibi çevrede hava kirliliği oluşturacak bir tesis bulunmamaktadır. Bölgenin rakımı 2000 m'nin üzerinde olduğundan yüksek bitkilerin varlığı görülmemekte ve alpin zon görülmektedir. Bölge yılın 6 ayı kar altındadır.

\section{2. Örnekleme ve Hazırlama}

Çalışma alanı olan Karagöl yaylası yılın 6 ayı kar altında olduğundan arazi çalışmaları yaz aylarında yapılmıştır. Bölgeye Haziran 2019 ve Eylül 2019 aylarında arazi gezileri düzenlenmiş ve bu geziler esnasında 17 tür karayosunu toplanmıştır. Çalışma alnının Türkiye'deki konumu Şekil 1'de ve Çalışma alanı haritası da şekil 2'de görülmektedir. Toplanan numunelerle ilgili, lokasyon ve habitat bilgi kayıtları tutularak polietilen poşetlere konuldu. Numuneler Laboratuvara getirilip mikroskopla incelendi ve (Smith, 2004) tarafindan yazılan The Moss Flora of Britain and Ireland, (Frey ve ark., 1985) tarafından yazılan DieMoos-undFarnpflanzenEuropas ve (Pedrotti, 2001), tarafından yazılan Flora deiMuschiD'Italia gibi karayosunu Flora kitapları yardımıyla numunelerin türleri belirlendi. Tablo 1'de çalışma alanı istasyonları ve toplanan numunelerin tür isimleri verilmektedir. 


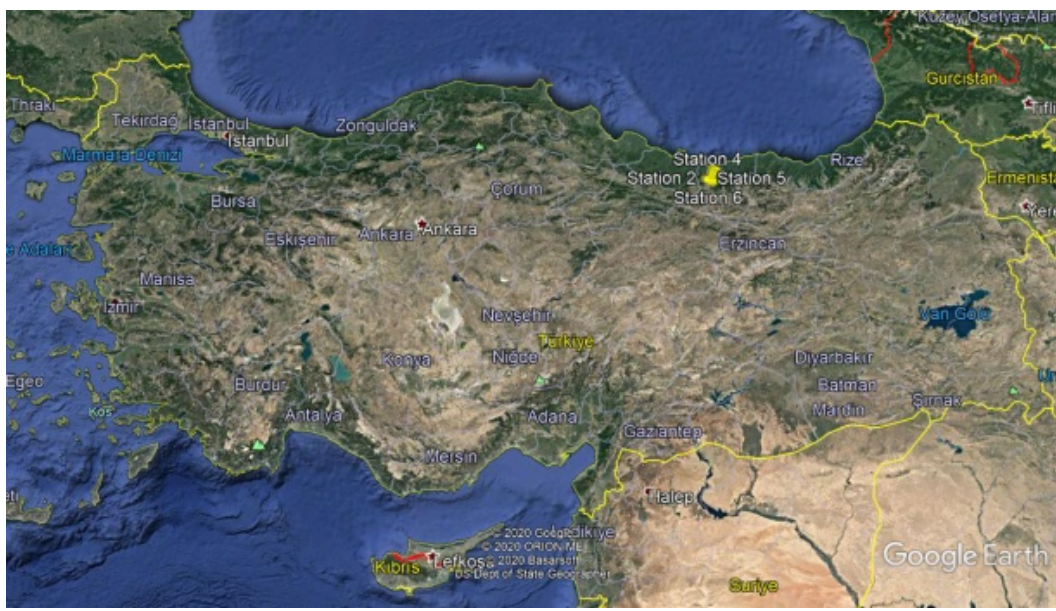

Şekil 1. Çalışma Alanı Türkiye haritası

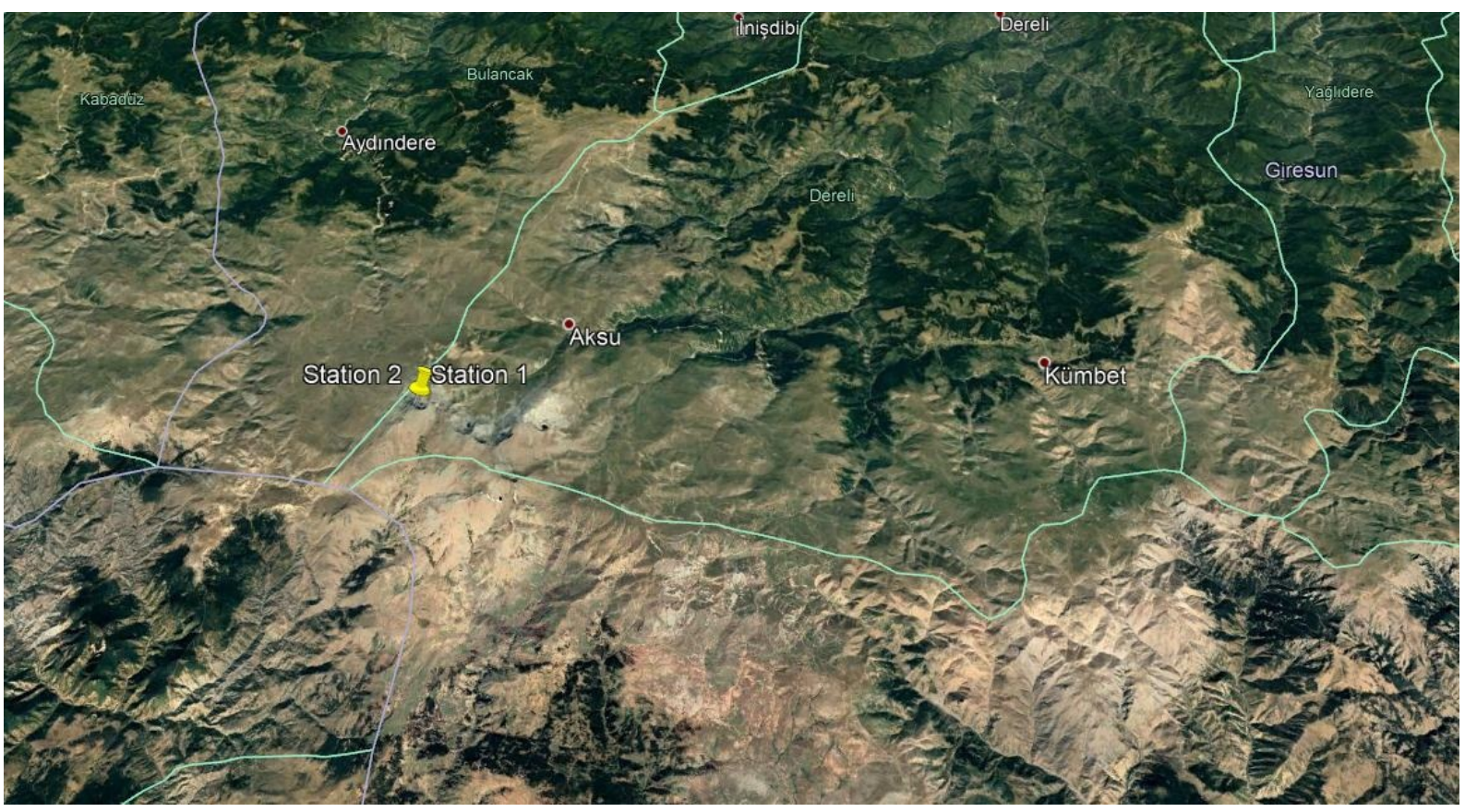

Şekil 2. Çalışma Alanı haritası 
Tablo 1. Giresun-Karagöl yaylası istasyonları ve Karayosunu Türleri

\begin{tabular}{|l|l|l|}
\hline $\begin{array}{l}\text { Örnek } \\
\text { No }\end{array}$ & İstasyon & Numune ismi \\
\hline 1 & İstasyon1 & Amblystegiumvarium(Hedw.) Lindb. \\
\hline 2 & İstasyon1 & Thuidiumtamariscinum(Hedw.) Schimp. \\
\hline 3 & İstasyon1 & HypnumcupressiformeHedw. \\
\hline 4 & İstasyon1 & Homalotheciumsericeum(Hedw.) Schimp. \\
\hline 5 & İstasyon1 & Eurhyncniumstriatum(Schreb. exHedw.) Schimp. \\
\hline 6 & İstasyon1 & Pseudoscleropodiumpurum (Hedw.) M.Fleisch. in Broth. \\
\hline 7 & İstasyon1 & Plagiotheciumsucculentum(Wils.) Lindb. \\
\hline 8 & İstasyon1 & Leucodonsciuroides(Hedw.) Schwaegr. \\
\hline 9 & İstasyon1 & GrimmiahartmaniiSchimp. \\
\hline 10 & & \\
\hline 11 & İstasyon1 & GrimmiadonniannaSm. \\
\hline 12 & İstasyon 2 & Hylocomiumsplenden(Hedw.) Schimp. \\
\hline 13 & İstasyon 2 & Ctenidiummolluscumvar.molluscum(Hedw.) Mitt. \\
\hline 14 & İstasyon 2 & Schistidiumapocarpum(Hedw.) Bruch\&Schimp \\
\hline 15 & İstasyon 2 & Eurhyncniumstriatulum(spruce) Schimp. \\
\hline 16 & İstasyon 2 & Oxyrrhynchiumhians(Hedw.)Loeske. \\
\hline 17 & İstasyon 2 & Brachytheciummildeanum(Schimp.) Schimp. \\
\hline & İstasyon 2 & BryumcapillareHedw. \\
\hline
\end{tabular}

\subsection{Inductively Coupled Plasma-Mass Spectroscopy (ICP-MS) Analizleri}

ICP-MS cihazında numuneleri analiz için hazırlarken bir gram numune alınıp mikrodalga çözme sisteminde $2 \mathrm{ml}$ hidrojen peroksit ve $6 \mathrm{ml}$ nitrik asit (CEM MARSX, 240/50, ABD) ile çözüldü. Bu çözelti daha sonra, distile edilmiş su ile 10 ml'lik balon jojede (Turkmen ve Dura, 2016) seyreltildi. Numuneler, analize gitmeden önce, $0.45 \mu \mathrm{m}$ 'lik bir filtreden geçirilerek süzüldü. Çok elemanlı standartlara göre (Merck, Darmstadt, Almanya) Kalibrasyon standartları hazırlandı. Geri kazanımların yüzdesi Ni için 93, Cu için 104, Cr için 113, Zn için 102, Cd için 91 ve Pb için 115 idi. Numuneler, mg.kg-1 yaş ağırlık olarak bir ICP-MS (BRUKER 820-MS, Almanya) kullanılarak Kurşun için üç kez analiz edildi (Turkmen ve Dura, 2016). Cihazın çalışma şartları Tablo 2'de verildi. 
Tablo 2. ICP-MS çalışma şartları

\begin{tabular}{|c|c|c|c|}
\hline Parameters & Settings & Parameters & Settings \\
\hline Plasmaflow & $18.0(1 / \mathrm{min})$ & Corner lens & -193 (volt) \\
\hline Auxiliaryflow & $1.80(1 / \mathrm{min})$ & Leftmirror lens & 45 (volt) \\
\hline Nebulizerflow & $0.90(1 / \mathrm{min})$ & Right mirror lens & 33 (volt) \\
\hline Sheathgas & $0.15(1 / \mathrm{min})$ & Bottommirror lens & 38 (volt) \\
\hline CRI gas $\mathrm{He}$ & $160(\mathrm{ml} / \mathrm{min})$ & Entrance lens & -1 (volt) \\
\hline CRI gas H2 & $100(\mathrm{ml} / \mathrm{min})$ & Fringebias & 2.5 (volt) \\
\hline RF power & $1.40(\mathrm{~kW})$ & Entranceplate & -39 (volt) \\
\hline Samplingdepth & $6.5(\mathrm{~mm})$ & Polebias & 0 (volt) \\
\hline Pump rate & $4(\mathrm{rpm})$ & Scanmode & Peakhopping \\
\hline Stabilizationdelay & $15(\mathrm{~s})$ & Dwell time & 20 (ms) \\
\hline Spraychamber & $3\left({ }^{\circ} \mathrm{C}\right)$ & Pointsperpeak & 1 \\
\hline First extraction lens & -1 (volt) & Scans/Replicate & 50 \\
\hline Second extraction lens & -180 (volt) & Replicates/Sample & 3 \\
\hline Third extraction lens & -226 (volt) & & \\
\hline
\end{tabular}

\section{Sonuçlar ve Tartışma}

Tablo 3'te Giresun Karagöl yaylasından toplanan karayosunu numunelerindeki kurşun konsantrasyonları verilmektedir. Buna göre; 17 tane numunenin ortalaması $14.47 \mathrm{mg} / \mathrm{kg}$ 'dır. Elde edilen bu ortalama Türkiye'de yapılmış çalışmaların kurşun ortalamalarının oldukça altındadır. Karayosunlarının yaprak yüzey alanları ile ağır metal absorplama kapasiteleri arasında bir ilişki olduğu Koz ve Cevik tarafından 2014 yılında yapılan çalışma ile ortaya konulmuştur. 
Tablo 3. Karagöl yaylası kurşun konsantrasyonları (mg/kg).

\begin{tabular}{|l|l|l|}
\hline Numune No & $\mathbf{P b}(\mathbf{m g} / \mathbf{k g})$ & Konum \\
\hline 1 & 14 & $40^{0} 2810^{\prime} \mathrm{K} ; 38^{0} 1701^{\prime} \mathrm{D}$ \\
\hline 2 & 12 & $40^{0} 2810^{\prime} \mathrm{K} ; 38^{0} 1701^{\prime} \mathrm{D}$ \\
\hline 3 & 19 & $40^{0} 2810^{\prime} \mathrm{K} ; 38^{0} 1701^{\prime} \mathrm{D}$ \\
\hline 4 & 21 & $40^{0} 2810^{\prime} \mathrm{K} ; 38^{0} 1701^{\prime} \mathrm{D}$ \\
\hline 5 & 15 & $40^{0} 2810^{\prime} \mathrm{K} ; 38^{0} 1701^{\prime} \mathrm{D}$ \\
\hline 6 & 21 & $40^{0} 2810^{\prime} \mathrm{K} ; 38^{0} 1701^{\prime} \mathrm{D}$ \\
\hline 7 & 15 & $40^{0} 2810^{\prime} \mathrm{K} ; 38^{0} 1701^{\prime} \mathrm{D}$ \\
\hline 8 & 6 & $40^{0} 2810^{\prime} \mathrm{K} ; 38^{0} 1701^{\prime} \mathrm{D}$ \\
\hline 9 & 14 & $40^{0} 2810^{\prime} \mathrm{K} ; 38^{0} 1701^{\prime} \mathrm{D}$ \\
\hline 10 & 11 & $40^{0} 281^{\prime} \mathrm{K} ; 38^{0} 1701^{\prime} \mathrm{D}$ \\
\hline 11 & 17 & $40^{0} 3004^{\prime} \mathrm{K} ; 38^{0} 2508^{\prime} \mathrm{D}$ \\
\hline 12 & 13 & $40^{0} 3004^{\prime} \mathrm{K} ; 38^{0} 2508^{\prime} \mathrm{D}$ \\
\hline 13 & 13 & $40^{0} 3004^{\prime} \mathrm{K} ; 38^{0} 2508^{\prime} \mathrm{D}$ \\
\hline 14 & 15 & $40^{0} 3004^{\prime} \mathrm{K} ; 38^{0} 2508^{\prime} \mathrm{D}$ \\
\hline 15 & 16 & $40^{0} 3004^{\prime} \mathrm{K} ; 38^{0} 2508^{\prime} \mathrm{D}$ \\
\hline 16 & 13 & $40^{0} 3004^{\prime} \mathrm{K} ; 38^{0} 2508^{\prime} \mathrm{D}$ \\
\hline 17 & 11 & $40^{0} 3004^{\prime} \mathrm{K} ; 38^{0} 2508^{\prime} \mathrm{D}$ \\
\hline Maksimum ve Minimum değerler & $\mathbf{2 1 - 6}$ & \\
\hline Ortalama Değer & $\mathbf{1 4 . 4 7}$ & \\
\hline & & \\
\hline
\end{tabular}

Tablo 4'te mevcut çalı̧̧̧adan elde edilen sonuçlar Dünya'daki ve Türkiye'deki bazı çalışmalardan elde edilen sonuçlarla kıyaslaması görülmektedir. Buna göre; Elde edilen sonuçlar, Finlandiya, Fransa, Almanya, Romanya, İtalya, İspanya, Hindistan'da yapılmış çalışmaların ortalamalarına göre yüksek olduğu görülmektedir. Yine aynı tabloda Türkiye'deki daha önce yapılmış çalı̧̧malarla kıyaslandığında 2008 yılında yapılmış çalışmadan düşük olduğu görülmektedir, fakat bu çalışma Türkiye'nin Doğu Karadeniz bölgesinin ana ulaşım hattı olan Sarp-Samsun sahil karayolu çevresindeki karayosunlarından elde edilen kurşun konsantrasyonu ortalamaları olduğu için düşüktür. Aynı tabloda, bu çalışma gibi insan kaynaklı kirlilikten uzak bölgeler olan kontrol bölgelerinden elde edilen verilerle kıyaslandığında ise benzer ortalamalar elde edildiği görülmektedir. Bu durumda bize, Giresun-Karagöl yaylasının kurşun kirliliği bakımından havasının son derece temiz olduğu sonucunu vermektedir. Fakat bununla birlikte karayollarının yaylaların en üst noktalarına 
kadar ulaşmış olması, kurşun kirliliği ve araç trafiği ile ilişkili, Al, V, Fe, Mn, Zn, Cu gibi ağır metal kirliliği tehlikesini arttırma potansiyelini de beraberinde getirmektedir.

Tablo 4. Literatür' deki verilerle mevcut çalışmanın kurşun konsantrasyon sonuçlarının kıyaslaması

\begin{tabular}{|l|l|l|}
\hline & $\mathbf{P b}(\mathbf{m g} / \mathbf{k g})$ & Kaynaklar \\
\hline Finlandiya & 3.0 & (Harmens, et al., 2007) \\
\hline Fransa & 5.7 & (Harmens, et al., 2007) \\
\hline Almanya & 4.6 & (Harmens, et al., 2007) \\
\hline Romanya & 14.4 & (Harmens, et al., 2007) \\
\hline Italya & 8.54 & (Loppi, et al., 1994) \\
\hline İspanya & 5.49 & (Fernandez, et al., 1992) \\
\hline Hindistan & 4.45 & (Bajpai, et al., 2010) \\
\hline Türkiye & 39.1 & (Koz, et al., 2008) \\
\hline Türkiye & 7.4 & Koz., v.d. 2012(Kontrol bölgesi) \\
\hline Türkiye & 22 & Koz., v.d. 2014(Kontrol bölgesi) \\
\hline Mevcut çalışma & 14.47 & \\
\hline
\end{tabular}

Avila-Perezve ark.'na göre, Pb gibi ağır metallerin yüksek konsantrasyonları insan sağlık riski bakımından son derece önemlidir. Araç trafiği kurşun kirliliği açısından önemli bir kaynaktır. Trafik kaynaklı ağır metaller, petrol, dizel motorlar, lastik aşınması, fren balataları, korozyon malzemesi gibi birçok faktörden çevreye verilmektedir (Thorpe ve Harrison, 2008).

(Di palma ve ark., 2017) kirli alanlardaki parçacıkların boyutu ile tarımsal, endüstriyel veya kentsel alanlar arasındaki ilişkiyi ortaya koydular. İzole edilmiş sanayi siteleri gibi oldukça kısıtlı alanlar dışında kirlilik kaynaklarını mekânsal olarak ayırt etmek oldukça zordur, çünkü havadaki birçok unsur, endüstriyel faaliyetler, tarımsal faaliyetler, egzoz dumanı, kentleşme kirlilikte etkili olabilir. Dur-kalk trafiği, trafikle ilişkili en önemli ağır metal kirlilik kaynaklarından biridir. (Westerlund ve Johansson, 2002)'a göre, alternatif olarak tünel kullanımına kıyasla Pb'nin dur-kalk ile ilişkili fren aşınması kirliliğinin 9 kat daha yüksek olduğu gösterilmiştir. (Harmens ve ark. 2007), Avrupa'da kurşun kirliliğinin yıllar içinde önemli ölçüde azaldığını gösterdiğini bildirdiler. Ancak Türkiye'deki kurşun konsantrasyonlarında aynı eğilim gözlenmedi.

Kurşun kirliliğinin en önemli sebeplerinden birisi kurşunlu benzin kullanımıdır. Kurşun kirliliği şehirleşme, insan nüfusunun yoğunluğu ve araç sayısı ile ilişkilidir. Hem çocuklarda hem de yetişkinlerde kurşun zehirlenmesi sinir sistemini etkiler. Yüksek miktarda kurşuna maruz kalırsa, çocuklarda ve yetişkinlerde beyne ve böbreklere zarar verebilir ve hatta ölüme bile neden olabilir (ATSDR, 2008). 
Farklı faktörlerden dolayı, karayosunlarındaki element birikimi konsantrasyonları değişebilir (Varela, ve ark., 2015). Diğer bir faktör de emisyon türüdür (gaz / partikül). Ayrıca karayosunu yapraklarının alan yüzeyleri ile element emilimi arasında da pozitif bir ilişki vardır (Koz ve Çevik, 2014). Vanderpoorten ve goffinet'e (2009) göre briyofitler, çevresel koşullara bağlı olarak morfolojik karakterlerinde geniş bir tür içi varyasyon sergiler. Susuzluk konsantrasyonları ile karşılaştırıldığında, analiz edilen karayosunu örneklerinde tespit edilen diğer elementlerin bolluğu düşük seviyelerden yüksek seviyelere kadar değişmektedir. Böyle bir varyasyon, yaprak yüzey alanı ve rüzgâr yönü, elemental konsantrasyonlar ve havadaki nem gibi diğer çevresel etkiler gibi karayosunlarının morfolojik ve anatomik yapısı ile doğrudan ilişkili olabilir (Koz, ve ark., 2008).

Kurşun, doğaya en fazla insan aktiviteleri sonucunda verilmektedir. Kurşun havaya kömür petrol ve atıkların yakılması esnasında verilmektedir. Kurşunlu benzin kullanımı, gelişmiş ülkelerde Türkiye'den önce yasaklandı ve Türkiye'de de kaldırıldı. Uzun süre yüksek oranda kurşunlu havaya maruz kalınırsa hem yetişkin hem de çocuklarda sinir sisteminde yıpranmalara ve bozulmalara sebep olur. Ayrıca kurşun dirsek, diz ve parmaklarda zayıflığa sebep olur. Yetişkinlerde kan basıncını biraz arttırmaktadır. Yetişkinlerde ve çocuklarda beyin ve böbrekte hasara neden olmaktadır. (URL-2)

\section{Sonuç}

Bu çalışmada, Giresun-Karagöl yaylasından toplanan karayosunu örnekleri ICP-MS ile analiz edilerek kurşun kirliliği hakkında bilgi edinilmiştir. Yapılan analizler sonucunda Giresun-Karagöl yaylasından toplanan karayosunlarında şehir merkezlerine göre Pb konsantrasyonunun düşük olduğu tespit edilmiştir. Giresun-Karagöl yaylasının havası kurşun konsantrasyonu açısından temiz olduğu fakat karayollarının yaylaların en üst noktalarına kadar ulaşmış olması sebebiyle potansiyel hava kirliliğine sebep olabileceği ihtimali doğurması tehlike arz etmektedir. Şehir merkezlerindeki kirliliğin, doğrudan nüfus yoğunluğu, kentleşme, trafik, tarımsal faaliyetler ile ilgilidir. Yapılan çalışma, karayosunlarının hava kirliliğinin belirlenmesinde önemli ve ucuz bir gösterge olduğunu göstermektedir.

\section{Araştırma ve Yayın Etiği Beyanı}

Yapılan çalışmada araştırma ve yayın etiğine uyulmuştur.

\section{Kaynaklar}

AgencyforToxicSubstancesandDiseaseRegistry (ATSDR), (2008). Division of Toxicology, Clifton Road, NE, Atlanta, GA, available at: http://www.atsdr.cdc.gov/toxprofiles/2008. 
Avila-Perez, P., Ortiz-Oliveros, H.B., Zarazua-Ortega, G., Tejeda-Vega, S., Villalva, A., Sanchez-Munoz, R., (2019). Determining of risk areasduetoexposuretoheavymetals in theTolucaValleyusingepiphyticmosses as a biomonitor. Journal of Environmental Management, 241, $138-148$.

Bajpai, R., Upreti, D.K., Nayaka, S., Kumari, B., (2010). Biodiversity, bioaccumulationandphysiologicalchanges in lichensgrowing in thevicinity of coalbasedthermalpowerplant of Raebarelidistrict, northIndia. Journal of HazardousMaterials, 174; 429-436.

Berg, T., Steinnes, E., (1997). Use of mosses (HylocomiumsplendensandPleuroziumschreberi) as biomonitors of heavy metal deposition: fromrelativetoabsolutevalues. EnvironmentalPollution, 98, 61-71.

DiPalma, A., Capozzi, F., Spagnuola, V.,Giordano, S., Adamo, P. (2017). Atmosphericparticulatematterinterceptedbymoss-bags: relationstomosstrace element uptakeandlanduse. Chemosphere, 176, 361-368.

Fernandez, M.A., Martinez, L., Segarea, M., Garcia, J.C., Espella, F., (1992). Behavior of heavymetals in thecombustiongases of urban wasteincinerators. EnvironmentalScience\& Technology, 26, 1040-1047.

Frey,W., Frahm, J. P., Fischer, E., Lobin,W. (1985) DieMoosundFarnpfanzenEuropas. Stuttgart: G. Fischer.

Grodzinska, K., Szarek-Lukaszewska, G., (2001). Response of mossestotheheavy metal deposition in Polandan overwiev. EnvironmentalPollution, 114, 443-451.

Harmens, H., Norris, D.A., Koerber, G.R., Buse , A., Steinnes, E., Ruhling, A. (2007). Temporaltrends in theconcentration of arsenic, chromium, copper, iron, nickel, vanadiumandzinc in mossesacross Europe between 1990 and 2000. Atmospheric Environment, 41, 6673-6687.

Jaradat, Q. M., Momani, K. A., (1999). Contamination of roadsidesoil, plantsandairwithheavymetals in Jordan, a comparativestudy. TurkishJournal of Chemistry, 23, 209-220.

Koz, B., Cevik, U., Ozdemir, T., Duran, C., Kaya, S., Gundogdu, A. andCelik, N., (2008) Analysis of mossesalong Sarp-Samsun highway in Turkey. Journal of HazardousMaterials, 153, 646-654.

Koz, B., (2014). Energydispersive X-ray fluorescenceanalysis of mossandsoilfromabandonedmining of $\mathrm{Pb}-$ Znores. EnvironmentalMonitoringandAssessment, 186, 5315-5326.

Koz, B., Cevik, U., (2014). Leadadsorptioncapacity of somemossspeciesusedforheavy metal analysis. EcologicalIndicators, 36, 491-494.

Loppi, S., Chiti, F., Corsini, A., Bernardi, L., (1994). Lichenbiomonitoring of tracemetals in thePistoriaarea (Italy). EnvironmentalMonitoringandAssessment, 29, 17-27.

Massadeh, A.M. Snook, R.D., (2002). Determination of $\mathrm{Pb}$ andCd in roaddustsovertheperiod in which $\mathrm{Pb}$ wasremovedfrom petrol in the UK. Journal of EnvironmentalMonitoring, 4, 567-572.

Pedrotti, C. C. (2001). Flora DeiMuschiD'Italia. Rome: Antonia DelfinoEditore.

Rühling, A., Tyler, G., (1968). An ecologicalapproachtothelead problem. BotaniskaNotiser 122, 248-342.

Smith, A.J.E. (2004). Themoss flora of Britain andIreland. Edinburgh: Cambridge UniversityPress.

Turkmen.M., Dura, N. (2016). Assessment of heavy metal concentrations in fishfromsouth western blacksea. IndianJournal of Geo-Marine Sciences, 45(11), 1552-1559.

Thorpe A., Harrison, R.M. (2008). Sourcesandproperties of non-exhoustparticulatematterfromroadtraffic: areview. Science of the Total Environment, 400, 270-282.

URL-1: http://www.ekonomihaber7.com (08.Mart.2018)

URL-2: http://www.epa.gov./lead-air-pollution. (26.Nisan.2021)

Westerlund, K.G., Johansson, C. (2002). Emissions of metalsandparticulatematterduetowear of brakelinings in Stockholm. In: Brebbia, C.A., Martin-Duque, J.F.(Eds), AirPollution X. WIT Press, Southampton; 793-802; 2002.

Varela, Z., Fernandez, J.A., Real, C., Carballeira, A., Aboal, J.R., (2015).Influence of thePhysicochemicalcharacteristics of pollutants on theiruptake in moss. Atmospheric Environment, 102, 130-135.

Vanderpoorten, A., Goffinet, B., (2009).Introductiontobryophytes. New York: Cambridge UniversityPress.

Wolterbeek, B.(2002). Biomonitoring of trace element airpollution: principles, possibilitiesandperspectives. EnvironmentalPollution, 120 (1), 11-21.

Zechmeister, H.G., Grodzinska, K. andSzarek-Lukaszewska, G. (2003). Bryophytes. In: Markert, B.A., Breure A.M. Zechmeister, H.G. (eds.): Bioindicators/Biomonitors (principles, assessment, concepts).Elsevier. Amsterdam, 339- 375. 